\title{
Melanocytic lesions: A pilot study using dual HMB-45/Ki67 staining with a new depth measurement to aid diagnosis
}

\author{
Darrell J Johnston, BSc Hons Biomedical Science, MSc, RLI \\ Phuoc-Tan Diep, MBChB, FRCPath, Consultant Histopathologist, RLI
}

\begin{abstract}
Diagnostic pitfalls have been widely documented on atypical naevi, a group of pigmented lesions that occupy a grey portion between benign and malignant variants. As malignant melanoma is associated with a high mortality rate it is vital we have a reliable diagnostic testing method for these challenging lesions. A pilot study was proposed using dual immunohistochemistry staining with a new depth measurement, the Lancaster depth, to develop a validating tool to measure difficult atypical lesions against. The Lancaster depth was recorded for 68 pigmented lesions; 34 benign and 34 malignant, where measurement was taken from the granular layer to the deepest HMB45/Ki67 dual stained melanocyte. This measurement was compared against the established Breslow depth measurement where the distance between the two was of interest. The mean malignant Lancaster depth at $1.51 \mathrm{~mm}$ was higher than that of the benign at $0.12 \mathrm{~mm}$. There was a statistically significant difference of $1.40 \mathrm{~mm}, P=<.001$. There was a statistically significant difference between the mean Lancaster and Breslow depths in both malignant and benign lesions. In the malignant group this was $0.28 \mathrm{~mm}$, $P=<.001$ compared to the benign which was $1.07 \mathrm{~mm}$, $P=<.001$. A narrow distance ratio between the two depths indicated a malignant diagnosis. A wider distance ratio was indicative of a benign diagnosis. As a diagnostic validator the Lancaster depth shows promises but has limitations as a standalone model. Working in conjunction with the Breslow depth we potentially have a useful adjunct to accurately diagnose difficult atypical lesions.
\end{abstract}

\section{KEYWORDS}

Melanocyte, naevi, melanoma, HMB-45, Ki67, Lancaster depth, Breslow depth.

\section{ABBREVIATIONS}

Malignant melanoma (MM), benign naevi (BN), haematoxylin and eosin (H\&E), atypical naevi (AN), benign compound naevi (BCN), Human Melanoma Black-45 (HMB-45) Lancaster Depth (LD), Breslow Depth (BD), confidence interval (CI).

\section{INTRODUCTION}

Malignant melanomas (MM) have the highest mortality rates for any skin cancer. In 2014 they accounted for 2,500 deaths within the United Kingdom (UK) which averages out as seven deaths daily. The incident rate for this disease is increasing with a $45 \%$ rise during the last ten years. ${ }^{1}$

Biologically, the cells of interest here are melanocytes which are capable of producing the pigment melanin. ${ }^{2}$ When normal cellular control and regulation is lost, melanocytes may give rise to a varied set of neoplasms, some of which can be differentiated based on their appearance, microscopic features and behaviour. ${ }^{3}$ These pigmented neoplasms or lesions could be described as belonging to the 'melanocytic spectrum'. This spectrum spans benign naevi (BN) at one end and $\mathrm{MM}$ at the other. ${ }^{4}$ For the most part $\mathrm{BN}$ are biologically stable with no malignant potential. ${ }^{5}$ On the other hand MM is an aggressive cancer with metastatic potential ${ }^{6}$ which is showing increasing trends in both incidence and mortality rates. ${ }^{7}$

Incident rates of melanoma have increased, but so too has public awareness and education of the disease. ${ }^{8}$ Early detection and surgical excision achieves a favourable outcome at the curable stages, ${ }^{9}$ hence why there has been an increased number of patients presenting with pigmented lesions for early, accurate assessment. ${ }^{10}$

Routine haematoxlin and eosin (H\&E) staining along with light microscopy is the current gold standard for differentiating pigmented lesions in the "melanocytic spectrum' looking at specific and distinct morphological entities. ${ }^{11}$ The most distinct morphological entity is nests of melanocytes that are present in both BN and MM. These nests are located in the epidermis and the dermis of the skin. In BN, the nests show one distinguishing feature. They mature with depth. Here the melanocytes undergo various morphological changes, in relation to cytoplasm and nucleus ratios, as they extend deeper into the dermis. This maturation is absent in MM. Another distinguishing feature between $\mathrm{BN}$ and $\mathrm{MM}$ is the size of the nests. In $\mathrm{BN}$ these become smaller as they extend into the dermis whereas the nest size in MM typically remains constant. ${ }^{12}$ Looking specifically for these features or absence of them allows $\mathrm{H} \& \mathrm{E}$ staining to be a relatively reproducible diagnosis to differentiate between $\mathrm{BN}$ and MM. ${ }^{11}$

As well as H\&E staining and light microscopy observation of features, MM cases are also incorporated with an important prognostic depth measurement known as the Breslow depth (BD) or Breslow thickness, ${ }^{13}$ particularly if the melanoma is in vertical growth phase (extending downwards into and beyond the dermis). When a melanoma is in this growth phase it has the potential to spread (metastasise) to other sites of the body. ${ }^{14}$ It is therefore extremely important to monitor this and the $\mathrm{BD}$ enables the physician to predict the chances of a patient developing metastases ${ }^{15}$ as well as allowing for planned therapies and treatment. ${ }^{16}$ Measured by ocular micrometer it is measured from the granular layer of the epidermis to the deepest point of tumour penetration. ${ }^{13}$

In the middle of the 'melanocytic spectrum' is a group of lesions known as atypical naevi (AN) though some publications refer to them as dysplastic naevi. For the purpose of this report they will be referred to as atypical naevi. AN could be described as residing in a 'grey zone' as they share many diagnostic characteristics with both $\mathrm{BN}$ and $\mathrm{MM},{ }^{17}$ sharing both physiological and behavioural 
attributes. ${ }^{18}$ These overlapping trajectories can lead to diagnostic difficulties during histological assessment and even subjective diagnoses. ${ }^{19}$ Over-calling an atypical lesion as malignant can result in inconvenient morbidity implications for the patient since an excision biopsy or wide local excision is normally elected upon diagnosis of melanoma. ${ }^{20}$ Of course, under-calling an atypical lesion as benign could have potentially terminal consequences. ${ }^{7}$ Litigation is also an important consideration since misdiagnosing of melanoma cases constitutes highly amongst medical malpractice claims. ${ }^{21}$

A definitive test is required to accurately differentiate this group from the other entities in the spectrum. ${ }^{7}$

Immunohistochemistry, a routine histological technique introduced in 1941 utilises specific antibodies to target and demonstrate antigens in tissue sections. When the antigen and antibody bind a colour reaction is visible down the light microscope. ${ }^{22}$ Dual immunohistochemistry enables us to take this one step further and view two antigens in one cell as two separate colours in a process known as co-localisation. ${ }^{23}$

Out of all the available immunohistochemistry markers in dermatology, S100 has been shown to be the most sensitive with regard to diagnosing melanocytic lesions. One major drawback with $\mathrm{S} 100$ is its lack of specificity. $^{24}$ Melan-A is another useful marker for differentiating melanocytic and non-melanocytic lesions, however it stains both benign and malignant melanocytic tumours. This renders it not suitable as a differentiator between $\mathrm{BN}$ and MM. ${ }^{25}$

Human Melanoma Black-45 (HMB-45), a cytoplasmic marker ${ }^{26}$ is specific for melanocytic lesions and has particular uses in detecting the maturation pattern expressed by benign melanocytic naevi. ${ }^{27}$ Recognising this maturation pattern enables the HMB-45 marker to differentiate $\mathrm{BN}$ from MM since expression is lessened as depth into the lesion increases in the benign variant. In contrast MM expression with HMB-45 is more consistent in the deeper aspects of the lesion. There are exceptions however, therefore HMB-45 expression or indeed lack thereof cannot be used solely as a leading or gold standard in differentiating benign and malignant melanocytic lesions. ${ }^{24}$

Ki67, a nuclear ${ }^{24}$ and cell proliferation marker shows positivity in less than $1 \%$ of cells in BN. Generally, any positivity is only shown in the superficial aspects of the dermis. Its pattern of expression highlights the presence or absence of maturation, showing reactivity in more than $10 \%$ of cells at the deep aspect of melanoma lesions. Put another way, if HMB-45 and Ki67 express a pattern that is dominant at the superficial aspect i.e. top of the lesion and absent in the deep aspect i.e. bottom of the lesion this will favour a diagnosis of a $\mathrm{BN}$ rather than $\mathrm{MM} .^{27}$

The primary aim of this study was to establish if there was a statistically significant difference in measurement depths of dual stained HMB-45:Ki67 melanocytes between $\mathrm{BN}$ and MM lesions so that we could have a potential validating tool to measure difficult atypical lesions against. A novel measurement, the 'Lancaster Depth' (LD) was designed to test this hypothesis.

\section{MATERIALS AND METHODS}

\section{Study Design}

This was a blinded, retrospective study on known positive reported cases from the University Hospitals of Morecambe Bay (UHMB) histopathology department's archives.

\section{Samples}

\section{Case Selection}

Searches were carried out via the electronic health record search programme Snomed CT ${ }^{\text {TM }}$ which defined the benign group as 'benign compound naevi' (BCN) and the malignant group as 'malignant melanoma' (MM). By default all samples with MM status were automatically in vertical growth phase. Paraffin blocks retrieved were sourced no earlier than 2015 since samples dated prior to this were no longer housed in the laboratory buildings.

\section{Lesion presence/suitability}

Paraffin blocks were randomised and a $4 \mu \mathrm{m}$ tissue section for each one was cut on a Thermo Shandon Finesse semiautomated rotary microtome, fixed to a glass slide and stained with H\&E. The specimen number for each block was recorded on each slide for traceability. The slide was also given a number for analysis i.e. Slide 1, 23 etc.

\section{Slide Analysis}

\section{Antibody Optimisation}

HMB-45 and Ki67 antibodies were already in routine diagnostic use within UHMB histopathology department.

\section{Dual immunohistochemistry staining protocols}

As dual immunohistochemistry was not in the diagnostic scope of practice for the laboratory, a new protocol had to be worked up where the Fast Red and diaminobenzidine (DAB) detection kits were allocated to the HMB-45 and Ki67 markers.

\section{Selection of control tissue}

Routine control tissue was selected for each marker; skin containing melanin and tonsil for HMB-45 and Ki67 respectively.

\section{Sample testing}

\section{Pilot study}

A pilot study on a total of 20 slides was conducted in the first instance (10 cases from each group). Descriptive statistics were collated from this initial data with the statistical package for the social sciences (SPSS version 24). A power calculation was performed where it was determined for the results to be statistically valid a total of 32 samples in each group were required. 


\section{Addition of Amplification Kit to HMB-45 staining}

Whilst the staining quality of the first round of slides was deemed acceptable, the staining intensity of the HMB45 was slightly weak in a small minority of slides. It was decided for the further staining runs an amplification kit would be added after HMB-45 antibody incubation to improve the signal intensity.

\section{Lancaster Depth Analysis}

The LD was measured by locating microscopically at $\mathrm{x}$ 10 magnification the piece of the lesion (assuming there were multiple pieces in the block) where the tumour extended the deepest (Figs. 1 and 2). The granular layer of the epidermis was then identified before measuring downwards to the deepest reactive dual stained HMB45/Ki67 melanocyte in the lesion (Fig. 3). Measurements were made using an ocular meter. It was necessary at times to use different magnifications to identify the dual stained cells.

\section{Lancaster/Breslow distance measurement analysis}

The BD for the malignant group was measured by locating microscopically the granular layer of the epidermis and measuring downwards into the dermis to the deepest nondual stained melanocyte (identified by a haematoxylin counterstain). The same process was carried out for the benign lesions. It should be noted that a BD is not routinely measured for benign lesions (since this depth is prognostic and associated with a malignant diagnosis).

\section{Statistical Analysis}

Results were expressed as mean \pm standard deviation for the independent samples t-test which was used to compare the mean LD in the benign and malignant groups.

\section{Immunohistochemistry dual stained illustrations}

Immunohistochemistry illustrations of stained slides were produced on the Nikon Coolscope ${ }^{\mathrm{TM}}$ digital microscope.

\section{RESULTS}

\section{Sample selection}

A total of 148 cases were observed by H\&E. Of these, 77 cases were deemed suitable for dual staining due to presence of a lesion. From these, 68 went forward for LD measurement analysis. This was due to some staining abnormalities relating to the Ki67 marker where the control tissue demonstrated no positive staining.

\section{Patient characteristics}

Samples representing the female population made up the majority of both groups as can be observed in Table 1 . The mean age of patients in the MM group was $61.82 \pm 19.09$ years in comparison to the mean age of $43.32 \pm 16.50$ years in the BCN group. This can also be observed in Table 1. (Results are displayed as mean \pm standard deviation).

\section{Lancaster Depth analysis}

34 malignant samples and 34 benign samples were analysed.

On inspection of the boxplot shown in Fig. 4, three outliers representing larger lesions were noted in the malignant group. Inspection of their values did not reveal them to be extreme and they were kept in the analysis. LD measurements for each tumour group were not normally distributed, as assessed by Shapiro-Wilk's test $(P<.05)$. The LD was higher in the malignant group at $1.51 \mathrm{~mm}$ $\pm 1.41 \mathrm{~mm}$ compared to the benign group at $0.12 \mathrm{~mm}$ $\pm 0.10 \mathrm{~mm}$ with a statistically significant difference of $1.40 \mathrm{~mm}(95 \% \mathrm{CI}, 0.90 \mathrm{~mm}$ to $1.89 \mathrm{~mm}), t(33)=5.763, P=$ $<.001$.

\section{Lancaster/Breslow distance measurement analysis}

A paired-samples t-test was used establish a mean difference in the distance between the LD and BD for the malignant group. The LD for the malignant group had a mean measurement of $1.51 \mathrm{~mm} \pm 1.41 \mathrm{~mm}$ compared to the mean $\mathrm{BD}$ which was $1.79 \mathrm{~mm} \pm 1.52 \mathrm{~mm}$. The mean distance in the malignant group between the two depths was $0.28 \mathrm{~mm}$ ( $95 \% \mathrm{CI}, 0.21 \mathrm{~mm}$ to $0.35 \mathrm{~mm}), t(33)=8.223$, $P=<.001$

A paired-samples t-test was also used for the benign group to ascertain the mean difference in distance between the $\mathrm{LD}$ and $\mathrm{BD}$. The $\mathrm{LD}$ for the benign group had a mean measurement of $0.12 \mathrm{~mm} \pm 0.10 \mathrm{~mm}$ compared to the $\mathrm{BD}$ which was $1.19 \mathrm{~mm} \pm 0.81 \mathrm{~mm}$. The mean distance in the benign group between the two depths was $1.07 \mathrm{~mm}(95 \%$ CI, $0.78 \mathrm{~mm}$ to $1.36 \mathrm{~mm}), t(33)=7.562, P=<.001$.

\section{Immunohistochemistry dual stained illustrations}

Fig. 1 demonstrates how the LD was measured in a MM lesion and its close proximity to the BD. Fig. 2 demonstrates the LD in a BCN lesion and its much further proximity to the Breslow depth. A x 40 magnification of a dual stained HMB-45/Ki67 melanocyte is shown in Fig. 3.

Mean Lancaster and Breslow depths as well as mean distance between both depths for the malignant and benign groups are summarised in Table 2. All LD and BD measurements for the 68 samples are shown on Table A1 in Appendix A.

\section{DISCUSSION}

Diagnostic challenges and pitfalls have been widely reported and documented through numerous studies on $\mathrm{AN},{ }^{28}$ a group of pigmented lesions that occupy a grey portion in the middle of the 'melanocytic spectrum'. ${ }^{29}$

To date, several studies have proposed various diagnostic models to meet the challenges associated with typing these lesions. The first, by Nielsen et al. in $2011^{30}$ employed the use of a semi-quantitative analysis together with a more involved quantitative analysis. Overall, results demonstrated HMB-45 and Ki67 as helpful adjuncts to differentiate benign and malignant melanocytic lesions. Further, more complicated analyses in the form of cell counts and proliferation indices between Ki67/MART1 
and HMB-45/MITF melanocytes were required to arrive at conclusive results. ${ }^{30}$

A subsequent study in 2015 by Uguen et $a l^{7}$ was carried out which adopted a p16/HMB-45/Ki67 scoring system with a view to finding a more reliable and accurate diagnostic tool for challenging atypical melanocytic lesions. The said markers were analysed as single entities in the first instance with later aspects of the study taking advantage of dual staining methodology. As single entities all markers demonstrated inconsistent results. Dual staining was utilised by combining p16 (a regulatory protein for the G1/S checkpoint of the cell cycle) with Ki67, and HMB-45 with Melan-A. A Ki67 proliferation index using a progressive five class scale also formed part of the study. A scoring system where all markers were assigned points was set up, an overall score of $<4$ indicating a diagnosis of naevi and a score of $>4$ indicating primary melanoma. Thus this testing method was found to have a sensitivity of $97.4 \%$ and a specificity of $97.3 \% .^{7}$

Whilst previous studies relied on dual staining applications their results were only produced when used in conjunction with more complicated analyses. We therefore proposed the Lancaster Depth, a novel, concise and reproducible measurement involving dual staining concepts with HMB-45 and Ki67. Keeping the diagnostic model relatively simple, our study took advantage of the maturation properties of $\mathrm{HMB}-45$ and $\mathrm{Ki} 67^{27}$ and incorporated these with a defined measurement. The LD study concentrated on analysing the two entities at either end of the melanocytic spectrum rather than the difficult atypical lesions residing in the middle. The aim here was to establish a pattern between those two opposites that would potentially allow for the implementation of a diagnostic validating tool for AN.

Approximately half of all MM diagnoses each year are diagnosed in people over 65 years of age. ${ }^{1}$ The patient characteristic data from our study summarised in Table 1 reflects this with a higher mean age in the MM group compared to the BCN group. While outliers were detected in the MM group it was decided unanimously not to exclude them since they represented larger lesions.

There was a statistically significant difference of $1.40 \mathrm{~mm}(95 \% \mathrm{CI}, 0.90 \mathrm{~mm}$ to $1.89 \mathrm{~mm}), t(33)=5.763, P=$ $<.001$ between the malignant and benign groups of lesions. Positive HMB-45/Ki67 staining was more prominent in the deeper aspects of all melanoma lesions tested and the superficial aspects of BCN specimens. Indeed, the absence of dermal staining was noted across all benign samples, some dual staining not extending beyond the granular layer (and thus a LD measurement of 0 was recorded). In the MM group most samples demonstrated positive dual staining in at least the dermal component with some extending into the subcutaneous fat.

The primary aim of the LD analysis was to ascertain a cut-off point against which a challenging atypical lesion could be typed as benign or malignant. The recorded mean LD for the malignant group of $1.51 \mathrm{~mm} \pm 1.41 \mathrm{~mm}$, (95\% CI, $1.02 \mathrm{~mm}$ to $2.00 \mathrm{~mm}$ ) was therefore of particular interest since it was established the deepest LDs correlated with the MM lesions. The $95 \%$ CI was not optimal since it indicates the true mean of malignant LD measurements is likely to reside between $1.02 \mathrm{~mm}$ and $2.00 \mathrm{~mm}$. Overall, this means that any lesion measuring $<1.02 \mathrm{~mm}$ can be typed as benign whereas a lesion measuring $>2.00 \mathrm{~mm}$ can be said to be malignant. So in effect there are two cutoff points rather than one, leaving a grey portion in the middle. If the LD of a given lesion falls within this grey portion, the testing methodology is limited.

We therefore proposed the addition of a second measurement depth that involved measuring from the granular layer to the deepest non dual-stained melanocyte thus measuring all of the lesion. This measurement depth was, by definition, the already established BD for both study populations. When observing the lesion in its entirety microscopically it was obvious that in the malignant group, the dual staining extended downward to take up most of the lesion. In the benign group the dual staining represented a much smaller portion, restricted to the superficial aspects at the top of the lesion. This can be observed in Figs. 2 and 3. The mean distance between the two measurement depths in the malignant group was $0.28 \mathrm{~mm}$ (95\% CI, $0.21 \mathrm{~mm}$ to $0.35 \mathrm{~mm}$ ), $P<.001$. The mean distance for the benign group was $1.07 \mathrm{~mm}(95 \%$ CI, $0.78 \mathrm{~mm}$ to $1.36 \mathrm{~mm}), P<.001$. The much narrower $95 \% \mathrm{CI}$ in contrast to the wider $95 \% \mathrm{CI}$ for the malignant LD was noted, indicating the Lancaster/Breslow distance measurement analysis was a more sensitive model. A small distance between the LD and BD confirmed a malignant diagnosis. By definition this analysis confirmed that the smaller the distance between the LD and BD the more likely the deepest dual stained cell observed was a non-maturing mitotic melanocyte, hence confirming malignancy.

Results from both the Lancaster depth analysis and the Lancaster/Breslow distance measurement analysis are summarised in Table 2 .

Results from this data, whilst not conclusive, are promising. The benefits are:

- An accurate result delivered from the offset could eliminate anxiety if found to be benign or could elicit faster treatment and therapy regimes if found to be malignant, thus increasing the patient's survival prospects.

- Even when we incorporate the LD with the second measurement the methodology is still accurate and reproducible. Immunohistochemistry testing methods are in the routine scope of practice for most laboratories, therefore adding the $\mathrm{LD}$ analysis into existing scopes will not incur any major additional costs.

- There will be less need to send difficult patient cases to specialist centres therefore saving money on referral and transport costs. Furthermore, the BD measurement has been carried out by pathologists for over four decades, ${ }^{15}$ so they already have the skill sets.

- Preservation of tissue is another advantage. Both the Lancaster and BD measurements can be analysed on the same slide. Two markers can be co-localised in the one cell, therefore there is less need for cutting additional tissue sections. 
To conclude, our results indicate HMB-45 and Ki67 markers are potentially capable, in a dual staining setting, of differentiating $\mathrm{MM}$ from $\mathrm{BCN}$ when incorporated with a new measurement depth with further benefit when incorporated with the Lancaster/Breslow distance measurement analysis. The consistency of our results show that the LD validating tool is a potentially reproducible test that with additional research and fine tuning could become available to all centres, specialist and routine, to improve the diagnostic accuracy of difficult lesions in the melanocytic spectrum.

Table 1: Basic patient characteristics of samples analysed

\begin{tabular}{lll}
\hline & Malignant Melanoma $(\mathbf{n}=34)$ & Benign Compound Naevi $(\mathbf{n}=34)$ \\
\hline Age (years) & $61.82 \pm 19.09$ & $43.32 \pm 16.50$ \\
Gender (male/female) & $14 / 20$ & $15 / 19$
\end{tabular}

Total sample size $(\mathrm{n}=68)$, malignant melanoma cases $(n=34)$ and benign compound naevi cases $(n=34)$. Values are expressed as mean \pm standard deviation with male to female ratios in both groups favouring the female population. The mean age (years) in the benign group was considerably lower compared to the malignant group.

Figure 1: Microscope image at $x 10$ magnification showing HMB-45/Ki67 dual staining for a malignant melanoma sample in vertical growth phase. The Lancaster Depth was measured by locating microscopically where the lesion was deepest at $\mathrm{x} 10$ magnification. The measurement was taken from the granular layer $\boldsymbol{\nabla}$ in the superficial aspect downwards into the epidermis and dermis to the deepest reactive dual stained $\mathrm{HMB}-45 / \mathrm{Ki} 67$ melanocyte in the lesion $\bullet$. Measurements were made using an ocular meter and measured in $\mathrm{mm}$. The Breslow depth measurement was taken from the granular layer $\boldsymbol{\nabla}$ to the deepest non-dual stained melanocyte in a haematoxylin counterstain 9 . The close proximity of the Breslow depth to the Lancaster depth in a malignant lesion is observed.

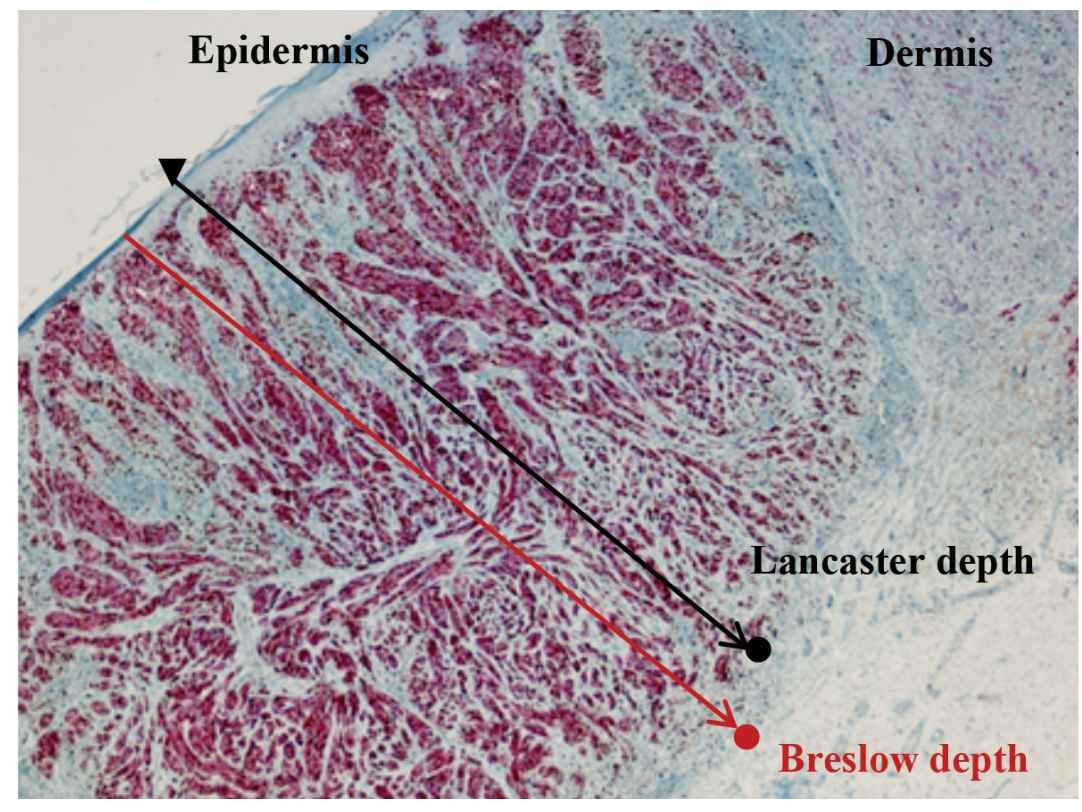

Figure 2: Microscope image at $x \quad 10$ magnification showing HMB-45/Ki67 dual staining for a benign compound naevus sample. The Lancaster depth measurement was taken by locating the deepest area of the lesion and measuring from the granular layer $\boldsymbol{\nabla}$ to the deepest dual stained melanocyte which did not extend beyond the epidermis o. The 'Breslow' depth measurement involved measuring from the granular layer $\boldsymbol{\nabla}$ to the deepest non-dual stained melanocyte $\bullet$. Measurements were made using an ocular meter and measured in $\mathrm{mm}$.

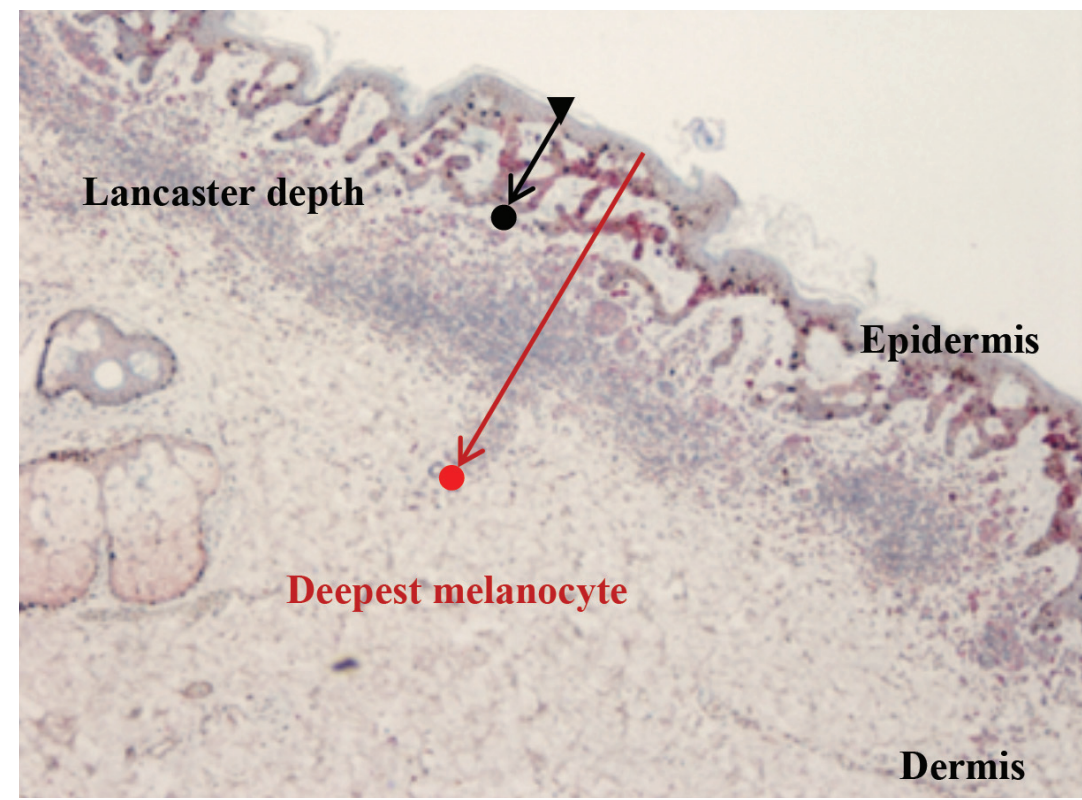


Figure 3: Microscope image at $x \quad 40$ magnification showing a dual stained HMB45/Ki67 melanocyte $\nabla$ surrounded by tumour negative melanocytes and tumour infiltrating lymphocytes $\boldsymbol{\Lambda}$.

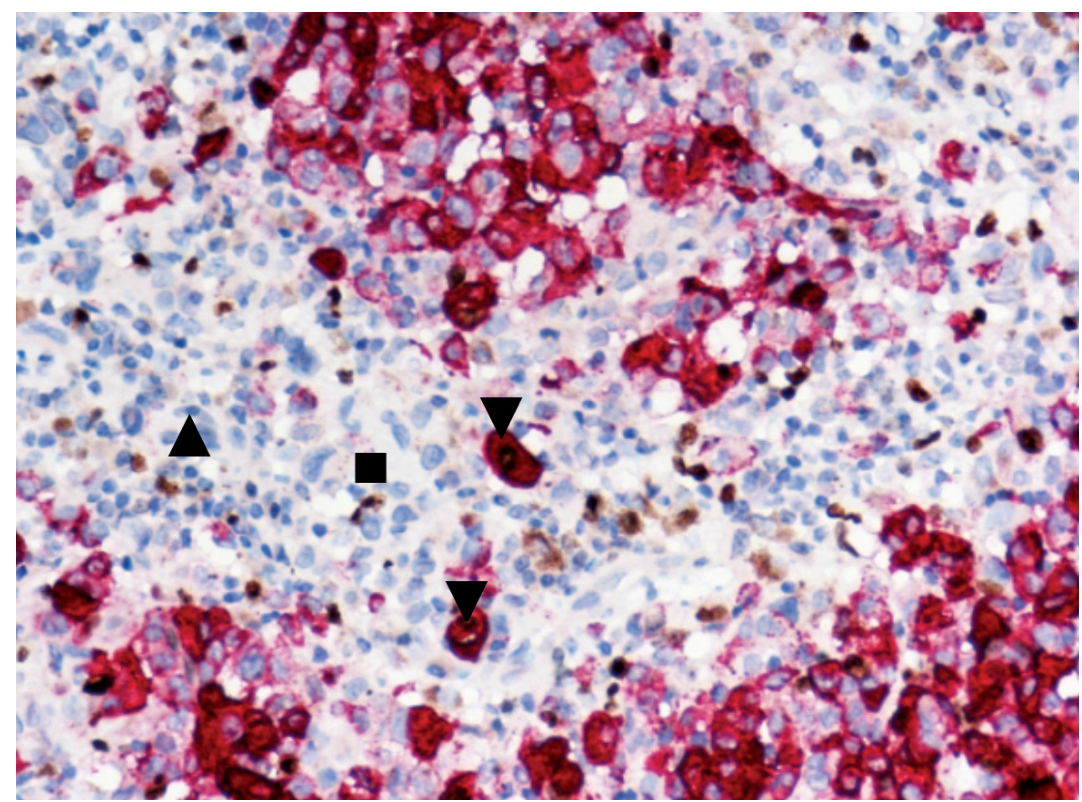

Figure 4: Box plot of the Lancaster depth of the benign and malignant melanocytic lesion groups with the minimum value, lower quartile, median, upper quartile and maximum values shown. Outliers are indicated with ${ }^{\circ}$. These represented larger lesions in the malignant group. They were not identified to be extreme and were kept in the analysis. A further study could eliminate these outliers by employing a larger sampling size and a higher number of larger lesions.

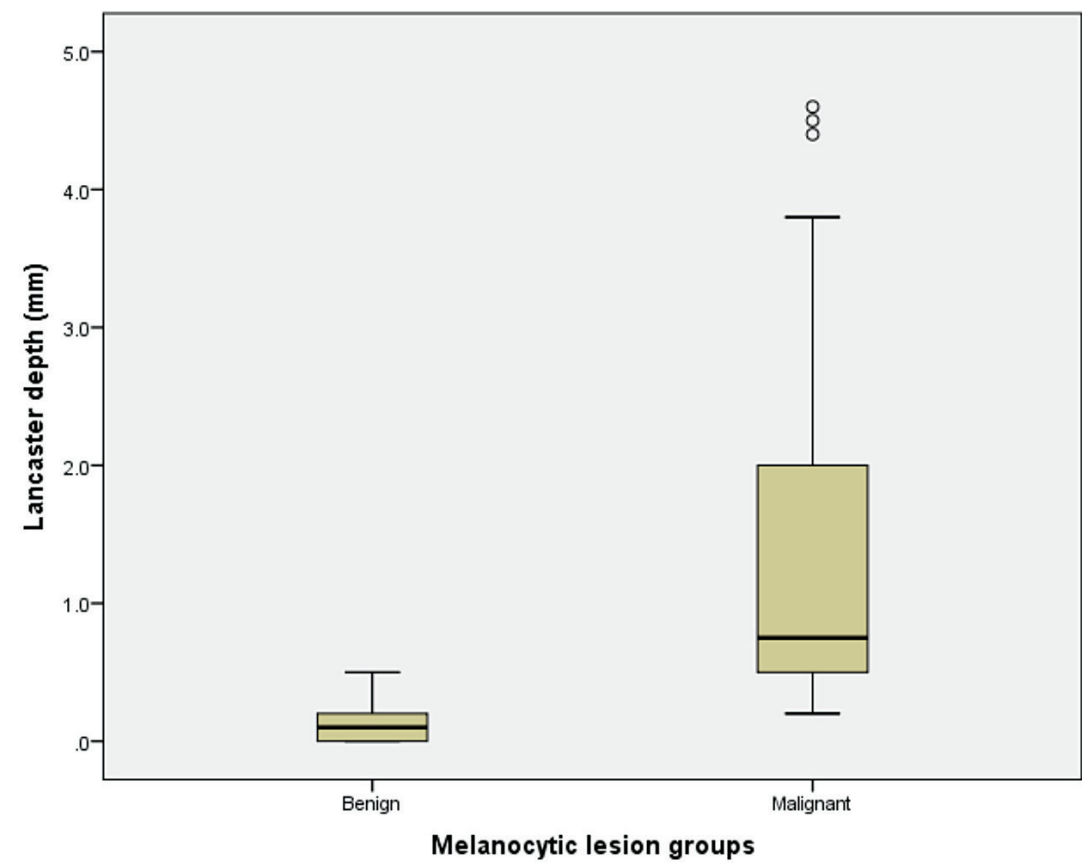

Table 2: MM and BCN Lancaster and Breslow depths and distance between

\begin{tabular}{lll}
\hline & Malignant Melanoma $(\mathbf{n}=34)$ & Benign Compound Naevi (n = 34) \\
\hline Lancaster depth (mm) & $1.51 \pm 1.41^{\star \wedge}$ & $0.12 \pm 0.10^{\star \mathrm{o}}$ \\
Breslow depth (mm) & $1.79 \pm 1.52^{\wedge}$ & $1.19 \pm 0.81^{\circ}$ \\
$\begin{array}{l}\text { Distance between } \\
\text { depths }(\mathrm{mm})\end{array}$ & $0.28^{\wedge}$ & $1.07^{\circ}$
\end{tabular}

The Lancaster depth defined as the deepest dual stained $\mathrm{HMB}-45 / \mathrm{Ki} 67$ reactive melanocyte in the lesion was measured against previously diagnosed tissue samples. Values, expressed as mean \pm standard deviation $(n=34)$ were higher in the malignant melanoma group compared to the benign compound naevi group, ${ }^{*} P=<.001$. In both tumour groups a second measurement, by definition the Breslow depth in the malignant group or the deepest melanocyte in the benign group was compared against the Lancaster depth with mean distance measurements reported, $\wedge^{\wedge} P=<.001$ and ${ }^{\circ} P=<.001$. 
Melanocytic lesions: A pilot study using dual HMB-45/Ki67 staining with a new depth measurement to aid diagnosis Darrell J Johnston, Phuoc-Tan Diep

\section{APPENDIX A}

Table A1: Lancaster and Breslow depth measurements for all samples

\begin{tabular}{|c|c|c|c|}
\hline $\begin{array}{l}\text { Slide } \\
\text { Number }\end{array}$ & \begin{tabular}{|l|} 
Lancaster \\
depth \\
$(\mathrm{mm})$
\end{tabular} & \begin{tabular}{|l} 
Breslow \\
depth \\
$(\mathrm{mm})$
\end{tabular} & Disease status \\
\hline 1 & 0.2 & 1.4 & Benign \\
\hline 2 & 0.2 & 0.5 & Benign \\
\hline 3 & 0.1 & 2.1 & Benign \\
\hline 4 & 2.0 & 2.3 & Malignant \\
\hline 5 & 0.6 & 0.8 & Malignant \\
\hline 6 & 0.1 & 0.8 & Benign \\
\hline 7 & 0.2 & 2.2 & Benign \\
\hline 8 & 0.1 & 0.6 & Benign \\
\hline 9 & 0 & 0.4 & Benign \\
\hline 10 & 0.4 & 0.6 & Malignant \\
\hline 11 & 2.3 & 2.8 & Malignant \\
\hline 12 & 1.2 & 1.4 & Malignant \\
\hline 13 & 0.1 & 2.5 & Benign \\
\hline 14 & 1.0 & 1.0 & Malignant \\
\hline 15 & 0.1 & 1.4 & Benign \\
\hline 16 & 0.2 & 2.6 & Benign \\
\hline 17 & 3.0 & 3.4 & Malignant \\
\hline 18 & 4.6 & 5.1 & Malignant \\
\hline 19 & 0.5 & 1.0 & Malignant \\
\hline 20 & 0.3 & 0.5 & Malignant \\
\hline 21 & 0.2 & 1.3 & Benign \\
\hline 22 & 0.1 & 1.0 & Benign \\
\hline 23 & 0.1 & 2.0 & Benign \\
\hline 24 & 1.9 & 2.0 & Malignant \\
\hline 25 & 4.4 & 4.9 & Malignant \\
\hline 26 & 0 & 0.5 & Benign \\
\hline 27 & 1.2 & 1.8 & Malignant \\
\hline 28 & 0.5 & 0.5 & Malignant \\
\hline 29 & 0.7 & 1.1 & Malignant \\
\hline 30 & 2.0 & 2.4 & Malignant \\
\hline 31 & 0.2 & 0.4 & Benign \\
\hline 32 & 0.2 & 0.5 & Benign \\
\hline 33 & 0 & 1.5 & Benign \\
\hline 34 & 0.1 & 2.4 & Benign \\
\hline 35 & 0 & 1.0 & Benign \\
\hline 36 & 0 & 0.4 & Benign \\
\hline 37 & 4.5 & 4.8 & Malignant \\
\hline
\end{tabular}

\begin{tabular}{|c|c|c|c|}
\hline $\begin{array}{l}\text { Slide } \\
\text { Number }\end{array}$ & $\begin{array}{l}\text { Lancaster } \\
\text { depth } \\
(\mathrm{mm})\end{array}$ & \begin{tabular}{|l|} 
Breslow \\
depth \\
$(\mathrm{mm})$
\end{tabular} & Disease status \\
\hline 38 & 1.2 & 1.6 & Malignant \\
\hline 39 & 2.0 & 2.3 & Malignant \\
\hline 40 & 0.1 & 0.5 & Benign \\
\hline 41 & 0 & 2.0 & Benign \\
\hline 42 & 0.6 & 0.6 & Malignant \\
\hline 43 & 0.5 & 1.0 & Benign \\
\hline 44 & 0.7 & 0.8 & Malignant \\
\hline 45 & 0.3 & 0.5 & Malignant \\
\hline 46 & 0.2 & 0.3 & Benign \\
\hline 47 & 0.2 & 0.4 & Malignant \\
\hline 48 & 0.2 & 0.4 & Benign \\
\hline 49 & 0.5 & 0.7 & Malignant \\
\hline 50 & 0.3 & 0.8 & Malignant \\
\hline 51 & 0.1 & 1.0 & Benign \\
\hline 52 & 0.5 & 0.5 & Malignant \\
\hline 53 & 0.1 & 0.7 & Benign \\
\hline 54 & 3.7 & 4.5 & Malignant \\
\hline 55 & 0.8 & 0.9 & Malignant \\
\hline 56 & 0.5 & 0.6 & Malignant \\
\hline 57 & 0.1 & 0.5 & Benign \\
\hline 58 & 0 & 2.5 & Benign \\
\hline 59 & 0.4 & 0.6 & Malignant \\
\hline 60 & 3.8 & 4.1 & Malignant \\
\hline 61 & 0.1 & 3.2 & Benign \\
\hline 62 & 3.8 & 4.1 & Malignant \\
\hline 63 & 0 & 0.7 & Benign \\
\hline 64 & 0.4 & 0.4 & Malignant \\
\hline 65 & 0.6 & 1.1 & Malignant \\
\hline 66 & 0.1 & 0.7 & Benign \\
\hline 67 & 0 & 0.6 & Benign \\
\hline 68 & 0.2 & 0.7 & Benign \\
\hline
\end{tabular}

\section{DECLARATIONS OF INTEREST}

There was no conflict of interest that could be perceived as prejudicing the impartiality of the research reported.

Correspondence to: phuoc-tan.diep@mbht.nhs.uk 


\section{REFERENCES}

1. Cancer Research UK (2017). Skin Cancer Mortality.

2. Cichorek M, Wachulska M, Stasiewicz A, Tymi A. Skin melanocytes: biology and development. Advances in Dermatology Allergology 2013;30(1):30-41.

3. Bastian BC. The molecular pathology of melanoma: an integrated taxonomy of melanocytic neoplasia. Annual Review of Pathology 2014;9:239-271.

4. Gundalli S, Kadadavar S, Singhania S, Kolekar R. Histopathological spectrum of benign melanocytic nevi our experience in a tertiary care centre. Our Dermatology Online 2016;7(1):21-25.

5. Haenssle HA, Mograby N, Ngassa A, Buhl T, Emmert S, Schon MP. Association of patient risk factors and frequency of nevus-associated cutaneous melanomas. JAMA Dermatology 2015; Nov 4:1-8.

6. Batus M, Waheed S, Ruby C, Petersen L, Bines SD, Kaufman HL. Optimal management of metastatic melanoma: current strategies and future directions. American Journal of Clinical Dermatology 2013;14(3):179-194.

7. Uguen A, Talagas M, Costa S. et al. A p16-Ki-67-HMB45 immunohistochemistry scoring system as an ancillary diagnostic tool in the diagnosis of melanoma. Diagnostic Pathology 2015;10:195.

8. Karimkhani C, Green AC, Nijsten T. et al. The global burden of melanoma: results from the Global Burden of Disease Study 2015. British Journal of Dermatology 2017;177(1):134-140.

9. Brehmer F, Ulrich M, Haenssle HA. Strategies for early recognition of cutaneous melanoma - present and future. Dermatology Practical \& Conceptual 2012;2(3).

10. Gilmore S. Melanoma screening: informing public health policy with quantitative modelling. PLoS ONE 2017;12(9).

11. Patterson JW, Hosler GA. Lentigines, nevi and melanomas. In: Patterson JW. Weedon's skin pathology, 4th ed. Oxford: Elsevier, 2016:888.

12. Calonje E, Brenn T, Lazar A, McKee PH. McKee's pathology of the skin: with clinical correlations. 4th ed. Oxford: Elsevier/Saunders, 2012.

13. Rebecca VW, Sondak VK, Smalley KSM. A brief history of melanoma: from mummies to mutations. Melanoma Research 2012;22(2):114-122.

14. Crowson AN, Magro CM, Mihm Jr MC. Prognosticators of melanoma, the melanoma report, and the sentinel lymph node. Modern Pathology 2006;19:S71-S87.

15. Rashed H, Flatman K, Bamford M, Teo KW, Saldanha G. Breslow density is a novel prognostic feature in cutaneous malignant melanoma. Histopathology 2017;70(2):264-272.

16. Marghoob AA, Koenig K, Bittencourt FV, Kopf AW, Bart RS. Breslow thickness and Clark level in melanoma. Cancer 2000;88(3):589-595.

17. Massi G, Leboit PE. Criteria for the diagnosis of nevus. Histological Diagnosis of Nevi and Melanoma. 2nd ed. Heidelberg: Springer, 2014:3.

18. Goldstein AM, Tucker MA. Dyplastic nevi and melanoma. Cancer Epidemiology, Biomarkers and Prevention 2013;22(4):528-532.

19. Balu M, Kelly KM, Zachary CB, et al. Distinguishing between benign and malignant melanocytic nevi by in vivo multiphoton microscopy. Cancer Research 2014;74(10):2688-2697.

20. Hieken TJ, Hernandez-Irizarry R, Boll JM, Coleman JEJ. Accuracy of diagnostic biopsy for cutaneous melanoma: implications for surgical oncologists. International Journal of Surgical Oncology 2013:196493.

21. Troxel DB. Pitfalls in the diagnosis of malignant melanoma: findings of a risk management panel study. The American Journal of Surgical Pathology 2003;27(9):1278-1283.

22. Ramos-Vara JA, Miller MA. When tissue antigens and antibodies get along: revisiting the technical aspects of immunohistochemistry - the red, brown and blue technique. Veterinary Pathology 2014;51(1):42-87.

23. van der Loos C (2009). User Protocol: practical guide to multiple staining.

24. Compton LA, Murphy GF, Lian CG. Diagnostic immunohistochemistry in cutaneous neoplasia: an update. Dermatopathology 2015;2(1):15-42.

25. Patrascu OM, Costache M, Dumitru AV, Mehotin CN, Sajin M, Lazaroiu AM. Expression of Bcl-2, Melan A and HMB-45 in Dysplastic Nevi. Maedica 2016;11(1):38-43.

26. Ohsie SJ, Sarantopoulos GP, Cochran AJ, Binder SW. Immunohistochemical characteristics of melanoma. Journal of Cutaneous Pathology 2008;35(5):433-444.

27. Prieto VG, Shea CR. Immunohistochemistry of melanocytic proliferations. Archives of Pathology \& Laboratory Medicine Online 2011;135:853-859.

28. Cockerell CJ, Tschen J, Evans B, et al. The influence of a gene expression signature on the diagnosis and recommended treatment of melanocytic tumors by dermatopathologists. Medicine 2016;95(40):e4887.

29. Reddy KK, Rogers GS. Management of dysplastic nevi: the role of complete surgical excision. The Melanoma Letter 2014;32(2).

30. Nielsen PS, Riber-Hansen R, Steiniche T. Immunohistochemical double stains against Ki67/ MART1 and HMB45/MITF: promising diagnostic tools in melanocytic lesions. The American Journal of Dermatopathology 2011;33(4):361-370. 